\title{
La professionnalisation des sages-femmes: analyse par la dynamique des représentations sociales et professionnelles
}

\section{Coralie Fregonese et Pierre Ratinaud}

Cette recherche porte sur la dynamique des représentations sociales et professionnelles intervenant dans le processus de professionnalisation des étudiants sages-femmes. Elle mobilise la théorie des représentations sociales et professionnelles et interroge un système représentationnel composé de deux objets: la profession de sage-femme et la profession de médecin.

Une étude transversale par questionnaires auprès de 358 étudiants à différents moments de la formation (de l'année du concours à la quatrième année en école) a mis en évidence l'évolution de ces représentations lors de la formation.

\section{Introduction}

La professionnalisation est au cœur des débats de l'enseignement supérieur en France (Bodin \& Orange, 2013; Gachassin, Labbé \& Mias, 2013; Gayraud, Simon-Zarca \& Soldano, 2011). En sciences de l'éducation, ce concept est notamment opérationnalisé dans des études qui s'intéressent aux transformations survenant lors des formations professionnalisantes (Lac, 2003). Dans cette perspective, l'une des approches possibles mobilise la théorie des représentations sociales et professionnelles. L'objectif est ici de d'étudier la dynamique des représentations qui intervient lors du processus de professionnalisation initié dans les formations. Il devient alors possible d'utiliser ces connaissances pour réinterroger l'ingénierie des formations. Nous tenterons de décrire, au travers de cette étude, comment la perception des professions de sage-femme et de médecin évolue chez les étudiants sage-femme durant leur parcours de formation.

La formation de sage-femme est une formation par l'alternance de cinq ans au cours desquels les étudiants font des apprentissages autant théoriques que pratiques. L'accès à l'école de sages-femmes se fait sur concours à l'issue d'une Première Année Commune en Santé (PACES) et se poursuit durant quatre années d'études spécifiques. Une enquête précédente (Frégonèse \& Ratinaud, 2015) sur les représentations professionnelles de la profession de sage-femme 
auprès des sages-femmes en activité nous a permis d'obtenir un point de référence sur la représentation de cette profession. Cette étude, menée cette fois auprès des étudiants, permet donc d'entrevoir comment cette représentation se met en place dans le déroulement de la formation.

Les objets professionnels visés sont la profession de sage-femme et la profession de médecin: le premier étant en lien direct avec la formation et le deuxième indissociable de l'exercice professionnel de la sage-femme. Dans les textes, la sage-femme est garante des situations physiologiques alors que le médecin est garant des situations pathologiques. Ces deux professions sont donc en interaction permanente. Cette enquête va tenter de décrire les transformations des représentations de ces deux professions au cours de la formation chez les étudiants. Cette transformation des représentations sera interprétée comme un marqueur du processus de professionnalisation.

Après avoir exposé les concepts de professionnalisation et de représentations sociales, nous présenterons les résultats obtenus par l'intermédiaire d'une enquête par questionnaire menée auprès des étudiants des 5 années de formation d'une école de sage-femme. Nous envisagerons alors les possibles aménagements de la formation potentiellement induits par ces résultats.

\section{Le processus de professionnalisation et la dynamique des représentations sociales et professionnelles}

\section{Le processus de professionnalisation}

La professionnalisation peut se définir comme un "processus de construction des connaissances, savoirs et identités reconnues comme faisant partie de la profession choisie» (Wittorski, 2007, p. 3). C'est aussi «un ensemble de processus en interaction conduisant à la construction et à l'évolution d'une identité professionnelle attestant de compétences spécifiques» (Lac, Mias, Labbé \& Bataille, 2010, pp. 135-136). La prise en compte des processus intervenant au niveau représentationnel permet de percevoir «la professionnalisation [...] comme le processus permettant la construction par les acteurs de connaissances, de savoirs professionnellement reconnus et opérants» (Lac \& al., 2010, p. 137).

La professionnalisation est un "«construit social», un enjeu et un objet social avant d'être un "construit scientifique», un enjeu et un objet de recherche» (Wittorski, 2010, p. 7). C'est un enjeu pour la formation afin de construire un dispositif adapté aux attentes de tous les acteurs: formateurs, professionnels et étudiants. La configuration de la formation par l'alternance, les aller-retour entre enseignement théorique et pratique réelle de l'activité, participe à l'évolution des représentations (Lac, 2003). Pour certains auteurs, cette alternance est un des dénominateurs communs des formations professionnalisantes (Maubant, Roger, Caselles-Desjardins, Mercier \& Gravel, 2010). Nous sommes bien ici 
dans le cadre de la professionnalisation-formation (Wittorski, 2012) avec le développement des savoirs et des compétences par les milieux de la formation et la recherche d'une efficacité et d'une légitimité plus grande des pratiques de formation.

Ainsi, apparaissent des expériences reposant sur une tentative d'articulation plus étroite entre l'acte de travail et l'acte de formation: il ne s'agit plus seulement de transmettre de façon déductive des contenus pratico-théoriques ou, au contraire, d'apprendre sur le tas (formation informelle) mais d'intégrer dans un même mouvement l'action au travail, l'analyse de la pratique professionnelle et l'expérimentation de nouvelles façons de travailler. Les logiques liées au recours à la formation changent, elles ne reposent plus tant sur l'adaptation de la main d'œuvre aux changements mais surtout sur l'élaboration et l'accompagnement de changements organisationnels. Cela engage un ancrage plus fort des actions de formation par rapport aux situations de travail (Wittorski, 2008, 2010). Par ailleurs, les professions elles-mêmes se modifient. Par exemple, la profession de sage-femme a vu son domaine de compétences s'élargir lors des réformes de 2009 et de 2015 en intégrant des actes de gynécologie jusqu'à présent réservés aux médecins. Ces réformes, ces changements au sein même des professions ou de leur contexte d'exercice ont des répercussions sur la façon dont les professionnels les perçoivent et sur les formations initiales de ces champs. Remoussenard (2010) souligne toutefois «l'inévitable décalage temporel» (p. 23) intervenant entre ces changements et leur prise en compte dans les formations.

Ratinaud \& Lac (2011) appuient alors sur le fait que la compréhension de la professionnalisation passe aussi par la compréhension des processus représentationnels qui se jouent au sein de la formation, ici, en école de sages-femmes. Connaître les représentations qui circulent dans la profession et la dynamique de celles mobilisées lors de la formation devient donc un enjeu pour maintenir l'adéquation entre objectifs de formation et attentes du terrain.

\section{Représentations sociales et professionnelles}

Moscovici (1976) définit une représentation sociale comme une forme de «savoir naïf» qui sert à «organiser les conduites et orienter les communications» d'un groupe social à propos d'un objet (p. 39). Jodelet (1991) précise que cette forme de connaissance est «socialement élaborée» (p. 53), c'est à dire construite et maintenue par les interactions communicationnelles entre les membres du groupe. Ces savoirs vont alors définir la réalité des objets sociaux pour ces groupes et donc permettre l'appropriation et la compréhension de l'environnement social et/ou professionnel par ses membres. De ce fait, elles vont fortement participer à définir et à maintenir les identités, tant sociales que professionnelles, et servir de guide pour les pratiques.

Les représentations professionnelles sont une catégorie particulière de représentations sociales. Elles portent sur des objets saillants dans les champs professionnels et sont spécifiquement partagées par les membres de la profession (Blin, 
1997; Piaser, 1999). La profession de sage-femme et la profession de médecin sont des objets à valeur d'enjeux sur lesquels les étudiants vont devoir se positionner et qui participeront à fonder leur identité professionnelle. Les interactions entre ces deux groupes professionnels et les communications qu'elles entraînent nous garantissent ici que nous avons bien à faire à des objets susceptibles de conduire à l'élaboration d'une image collective (Moliner, Rateau \& Cohen-Scali, 2002).

Pour Piaser \& Ratinaud (2010), les recherches sur les représentations professionnelles sont un enjeu pour les formations car «l'étude de l'écart entre la façon dont les professionnels perçoivent leurs objets de pratique et la façon dont ils sont enseignés est [...] un outil de pilotage des systèmes de formation» (p. 12).

La modélisation proposée par Bataille (2000, p.187) schématise les stades de transformation d'une représentation sociale en représentation professionnelle et précise la place des représentations préprofessionnelles (Bataille, 2007). Cette notion est le prolongement de celle de représentations socio-professionnelle» proposée par Fraysse (1996). Pour Piaser \& Bataille (2011), si cette représentation n'est plus uniquement 'sociale' car empreinte de bons nombres d'éléments techniques appartenant à une profession, elle n'est pas encore 'professionnelle', car insuffisamment constituée d'éléments expérientiels relevant de la mémoire du groupe professionnel considéré» (p. 49).

Pour certains auteurs (Michalot \& Siméone, 2013; Piaser \& Ratinaud, 2010), ces représentations, étapes intermédiaires entre représentations sociales et représentations professionnelles, sont le marqueur du processus de professionnalisation qui intervient dans les «situations relevant de l'une au moins de deux familles: la formation professionnelle et le travail en situation réelle» (Piaser \& Ratinaud, 2010, p. 11).

Pour approcher le domaine des représentations, nous avons privilégié une approche structurale. Abric (2003) décrit une représentation comme étant une «une organisation signifiante» (p. 13) autour d'un noyau central. C'est ce noyau central qui, pour lui, donne à une représentation sa signification. Autour de ce noyau central va s'organiser le système périphérique, plus riche en éléments mais moins stable et susceptible d'adaptation aux variations de contexte rencontrées par les acteurs. Dans ce cadre théorique, on considère qu'il y a un changement de représentation uniquement lorsque l'un des éléments du noyau central est modifié (Abric, 2003). La plupart du temps, le changement d'une représentation sociale est limité à une modification du "plus petit nombre possible d'éléments de la représentation initiale» (Abric, 1987, p. 74) concernant seulement la transformation de certains éléments «annexes (périphériques) composant la représentation, sans toucher aux éléments du noyau central» (Abric, 1987, p. 74).

Les éléments théoriques présentés permettent alors de souligner la pertinence d'une approche des marqueurs du processus de professionnalisation par l'observation des potentielles transformations des représentations sociales. 


\section{Matériel et méthode}

Afin de mettre en évidence la dynamique des représentations des professions de sage-femme et de médecin qui intervient dans la phase de formation des étudiants sages-femmes, nous avons réalisé une étude transversale sur les 5 années de formation au sein d'une même université.

Une première étude sur la connaissance des représentations professionnelles de la profession de sage-femme par les acteurs de la périnatalité (c'est-à-dire sagesfemmes et médecins) avait permis de comprendre les différentes perceptions de la profession de sage-femme au sein de professionnels travaillant en équipe ou en réseau et, au travers de ces perceptions, une forme avancée du processus de professionnalisation (Frégonèse \& Ratinaud, 2015). Ainsi pour la formation, cette enquête avait permis de mettre en évidence la perception technique de la profession par les médecins et la perception émotionnelle de la profession par les sages-femmes: les attentes de chacun vis-à-vis des étudiants divergent lors des périodes de stage ce qui est un poids dans la formation et ajoute des difficultés au positionnement des étudiants en stage.

Pour interroger l'ensemble des étudiants des cinq années de formation, nous avons utilisé un questionnaire, outil classique dans l'étude des représentations sociales (Abric, 2003). Ce questionnaire, dont la passation a eu lieu en octobre, contenait, entre autres, un test d'association libre (ou évocation hiérarchisée) sur les objets "profession de sage-femme» et "profession de médecin» et un test de centralité sur l'objet "profession de sage-femme». Pour les étudiants de première année, le recueil a été réalisé lors de sessions de colle de la PACES. Pour les quatre autres années, nous avons profité de notre fonction de formatrice pour remettre le questionnaire en main propre aux étudiants.

L'association libre consiste à demander à notre échantillon de citer 4 à 5 mots concernant ces objets. Par exemple, pour la profession de sage-femme, nous avons utilisé la formulation suivante: "Quels sont les 4 ou 5 mots ou expressions qui vous viennent spontanément à l'esprit lorsqu'on évoque la profession de sage-femme?».

Pour traiter ces données, la première opération a consisté en une catégorisation de l'ensemble des mots recueillis, de manière à ce que chaque catégorie soit identique entre les deux objets testés lorsque cela était possible.

Nous avons utilisé l'analyse de similitude (Degenne \& Vergès, 1973; Flament, 1962) pour traiter ces données. Une représentation sociale étant «une organisation, un ensemble de relations entre [des] éléments» (Abric, 2005, p. 222), elles peuvent être schématisées sous la forme d'un graphe qui lie par des "arêtes» les catégories conjointement exprimées par les sujets. Pour simplifier la lecture de ces graphes, nous passons par l'intermédiaire d'un arbre maximum. Dans la théorie des graphes, un arbre est «un graphe connexe et sans cycle» (Ratinaud, 2003). L'arbre maximum est le plus lourd (en termes d'information) de tous les arbres possibles. Nous utiliserons ici le pourcentage de cooccurrences comme 
indice pour mesurer la force des liens entre les catégories, ce qui correspond au pourcentage de sujets ayant associé les deux termes.

En phase de stabilité, l'identification des éléments centraux de la représentation étudiée est primordiale pour saisir le sens que le groupe attribue à l'objet, les individus étant dans l'impossibilité d'envisager l'objet sans ses caractéristiques centrales (Moliner, 2001). Parmi les méthodes proposées dans la littérature, nous avons choisi le test d'indépendance au contexte qui cherche à vérifier la centralité d'un item en testant son lien systématique (indépendamment du contexte) à l'objet (Lo Monaco, Lheureux \& Halimi-Falkowicz, 2008). Ce test a pu être mis en place grâce à l'étude précédente des représentations professionnelles de la profession de sage-femme qui avait permis d'obtenir des items appartenant potentiellement à la zone du noyau central. Les modalités de passage de ce test sont les suivantes: un item sur la profession est proposé, comme par exemple l'item accompagnement dans l'affirmation suivante: «une sage-femme accompagne toujours, dans tous les cas, ses patientes». Quatre modalités de réponses sont proposées: "certainement non", "plutôt non», "certainement oui», "plutôt oui». Un item est considéré comme central lorsque l'indépendance au contexte (réponse "certainement oui» et "plutôt oui») est attestée par une part de la population qui n'est pas significativement différente des $100 \%$. Le test de Kolmogorov-Smirnov est utilisé pour vérifier ce critère (Moliner, 2001, p. 265).

Pour l'ensemble de nos traitements, nous avons utilisé les logiciels R (R Development Core Team, 2014) et IRaMuTeQ (Ratinaud, 2014).

\section{Résultats}

L'enquête a été réalisée dans une des trente-cinq écoles de sages-femmes de France. Sur 566 questionnaires distribués aux étudiants des 5 années de formation, 358 questionnaires ont été exploitables: 232 réponses provenant de la PACES, 32 des étudiants de $2^{\mathrm{e}}$ année, 30 des étudiants de $3^{\mathrm{e}}$ année, 31 des étudiants de $4^{\mathrm{e}}$ année et 33 des étudiants de $5^{\mathrm{e}}$ année soit 126 réponses pour les quatre années de formation en école ( $100 \%$ de l'effectif de cette école).

Le tableau 1 rend compte de ces effectifs, des moyennes des âges et des répartitions par genre de ces années de formation.

Tableau 1: Effectifs, âge moyen et répartition en genre par année.

\begin{tabular}{|l|c|c|c|}
\hline \multicolumn{1}{|c|}{ Année } & Effectifs & Age (moyenne) & $\begin{array}{c}\text { Genre (femme/ } \\
\text { homme) en \% }\end{array}$ \\
\hline PACES (1e année) & 232 & $18,6[16-41]$ & $70 \% / 30 \%$ \\
\hline 2e année & 32 & $20[19-25]$ & $98 \% / 2 \%$ \\
\hline 3e année & 30 & $21,5[19-33]$ & $98 \% / 2 \%$ \\
\hline 4e année & 31 & $22[20-37]$ & $100 \% / 0 \%$ \\
\hline 5e année & 33 & $23,5[21-38]$ & $98 \% / 2 \%$ \\
\hline
\end{tabular}




\section{Évolution des résultats de l'association Libre}

Que ce soient les analyses réalisées sur la profession de sage-femme ou celles sur la profession de médecin, nous avons pu mettre en évidence les évolutions des représentations au cours du cursus de formation.

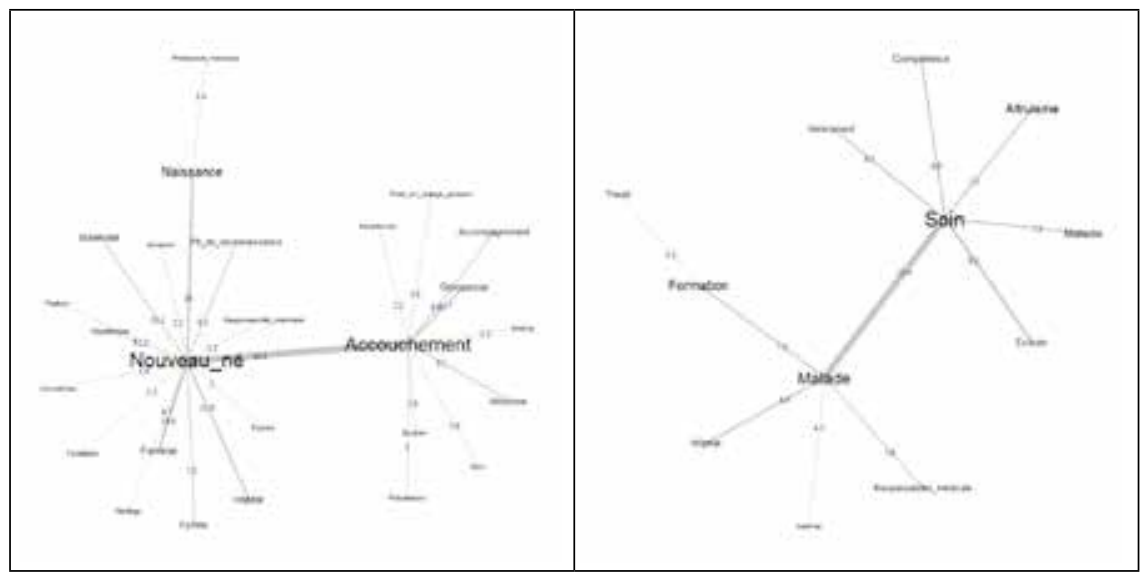

Graphiques 1 et 2: Arbre maximum des analyses de similitude sur les items apparaissant chez au moins $5 \%$ de la population de le année pour l'objet "profession sage-femme» à gauche et "profession médecin» à droite $(N=232)$.

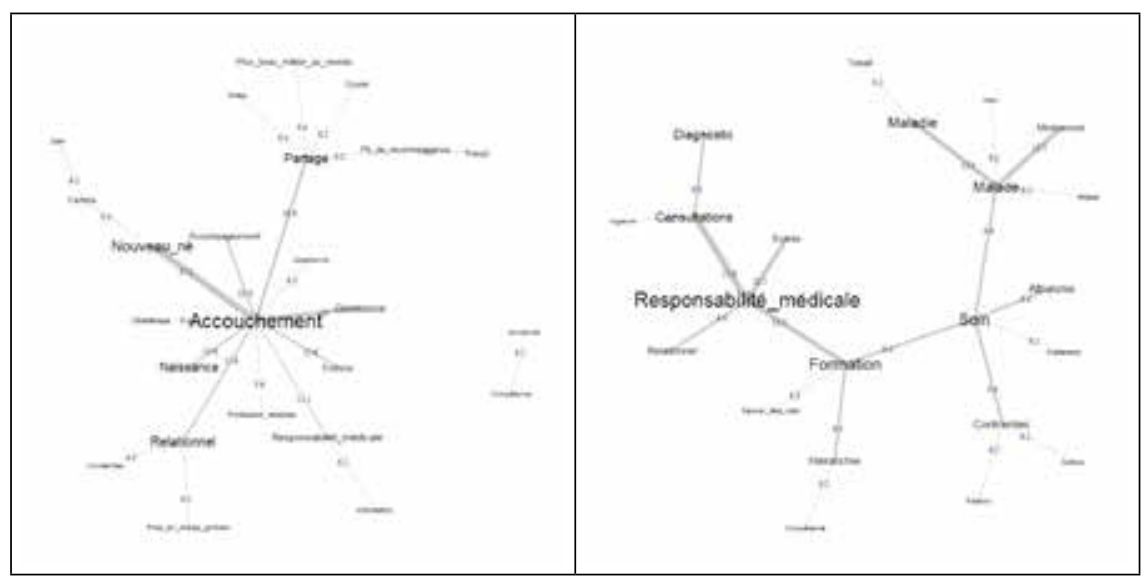

Graphiques 3 et 4: Arbre maximum des analyses de similitude sur les items apparaissant chez au moins $5 \%$ de la population de $2 e$ année pour l'objet "profession sage-femme» à gauche et "profession médecin» à droite $(N=32)$. 


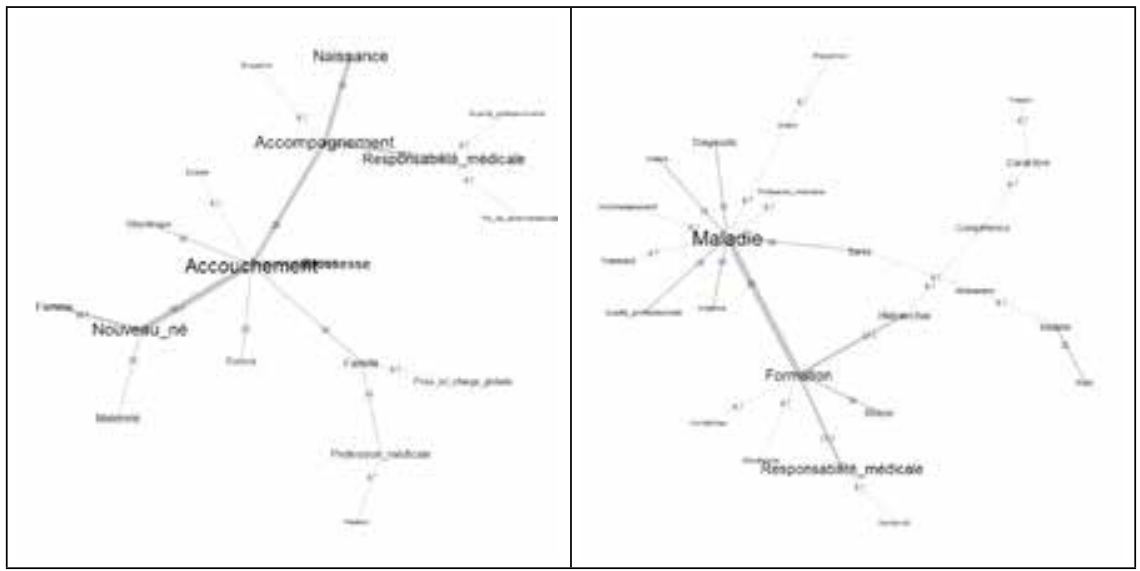

Graphiques 5 et 6: Arbre maximum des analyses de similitude sur les items apparaissant chez au moins $5 \%$ de la population de $3 e$ année pour l'objet "profession sage-femme» à gauche et "profession médecin" à droite $(N=30)$.

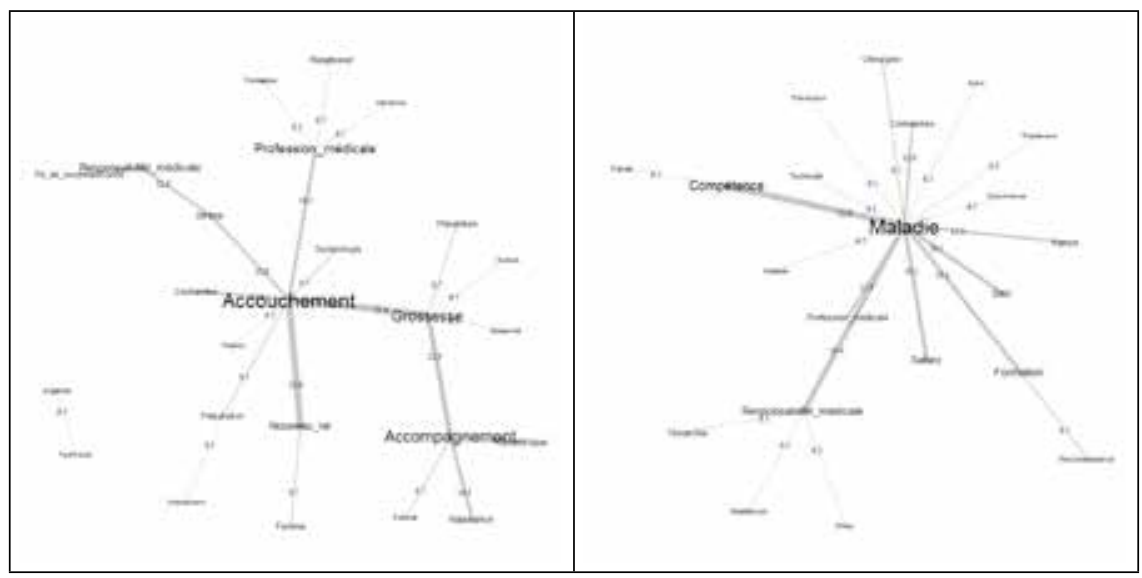

Graphiques 7 et 8: Arbre maximum des analyses de similitude sur les items apparaissant chez au moins $5 \%$ de la population de 4e année pour l'objet "profession sage-femme» à gauche et "profession médecin» à droite $(N=31)$. 


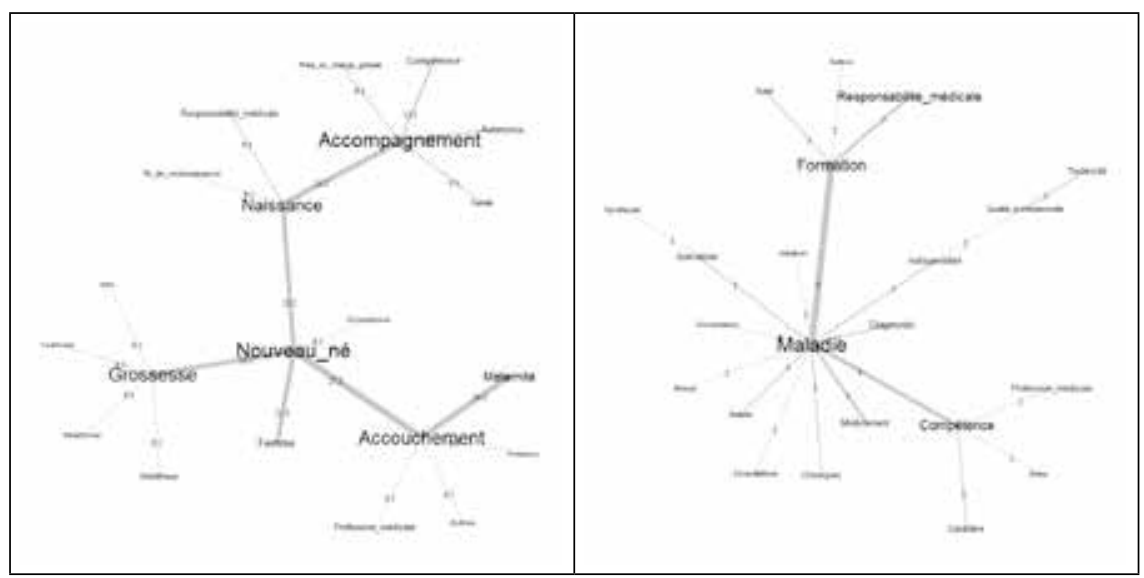

Graphiques 9 et 10: Arbre maximum des analyses de similitude sur les items apparaissant chez au moins $5 \%$ de la population de $5 e$ année pour l'objet "profession sage-femme» à gauche et "profession médecin» à droite $(N=33)$.

Les graphiques de similitude de 1 à 10 rendent compte de l'évolution de la représentation des deux objets concernés entre la première et la dernière année de formation. Ils mettent assez clairement en évidence le passage d'une lecture «naïve» (sociale) de ces professions à une lecture plus «armée» et plus professionnelle. Chez les étudiants de PACES, la représentation de la profession de sage-femme s'organise strictement autour des notions de nouveau-né et d'accouchement et celle de médecin autour des termes soin et malade. Dès l'entrée en école de sage-femme, les représentations se restructurent et se complexifient. Les changements significatifs dans l'évocation de ces items entre les années sont résumés dans le tableau 2 pour l'objet profession de sage-femme et dans le tableau 3 pour l'objet profession de médecin.

Tableau 2: items de l'objet "profession de sage-femme» présentant des différences significatives entre les années de formation; les «+» indiquent une sur-représentation significative de l'item pour l'année concernée, les "-» signalent une sous-représentation significative de l'item pour l'année concernée.

\begin{tabular}{|l|l|c|c|c|c|c|}
\hline Item & $\begin{array}{l}\text { Chi } 2 \text { et seuil de } \\
\text { significativité }(\mathrm{ddl}=4)\end{array}$ & $\begin{array}{c}1 \mathrm{e} \\
\text { année }\end{array}$ & $\begin{array}{c}2 \mathrm{e} \\
\text { année }\end{array}$ & $\begin{array}{c}3 \mathrm{e} \\
\text { année }\end{array}$ & $\begin{array}{c}4 \mathrm{e} \\
\text { année }\end{array}$ & $\begin{array}{c}5 \mathrm{e} \\
\text { année }\end{array}$ \\
\hline Nouveau-né & Chi2 $=26,84 ; \mathrm{p}<0.0001$ & + & - & - & - & - \\
\hline Relationnel & Chi2 $=42,75 ; \mathrm{p}<0.0001$ & & + & - & + & \\
\hline Profession médicale & Chi2 $=25,43 ; \mathrm{p}<0.0001$ & - & & + & + & \\
\hline Responsabilité médicale & Chi2 $=34,92 ; \mathrm{p}<0.0001$ & - & + & + & + & \\
\hline Autonomie & Chi2 $=30,44 ; \mathrm{p}<0.0001$ & - & & & & + \\
\hline Stress & Chi2 $=23,19 ; \mathrm{p}<0.0001$ & - & & & + & \\
\hline Technicité & Chi2 $=17,17 ; \mathrm{p}=0.001$ & - & & + & + & + \\
\hline
\end{tabular}


Concernant la profession de sage-femme, nous observons une évolution des termes cités lors de l'association libre. Le terme nouveau-né est surtout prégnant chez les premières années. La responsabilité médicale et le relationnel prennent de l'importance à partir de la $2^{\mathrm{e}}$ année; la technicité en $4^{\mathrm{e}}$ et $5^{\mathrm{e}}$ année alors que les notions de stress et d'autonomie sont plus présents en $4^{\mathrm{e}}$ année et celle de compétence en $5^{\mathrm{e}}$ année, dans un contexte préprofessionnel. Nous constatons également un décalage entre les représentations des étudiants de $5^{\mathrm{e}}$ année et les résultats que nous avons obtenus sur les professionnelles (Frégonèse \& Ratinaud, 2015). Les termes accouchement et accompagnement semblent avoir la même importance chez les étudiants alors que chez les professionnelles sages-femmes prédomine le terme accompagnement. Nous pouvons donc faire l'hypothèse que d'un point de vu représentationnel, la professionnalisation n'est pas achevée en $5^{\mathrm{e}}$ année. Le processus de professionnalisation ne s'interrompt donc pas à la sortie de l'école et se poursuit par l'acquisition de l'expérience professionnelle (Bataille, 2000).

Tableau 3: items de l'objet "profession de médecin" présentant des différences significatives entre les années de formation; les "+» indiquent une sur-représentation significative de l'item pour l'année concernée, les «-» signalent une sous-représentation significative de l'item pour l'année concernée.

\begin{tabular}{|l|l|c|c|c|c|c|}
\hline Item & $\begin{array}{l}\text { Chi } 2 \text { et seuil de } \\
\text { significativité }(\mathrm{ddl}=4)\end{array}$ & $\begin{array}{c}1 \mathrm{e} \\
\text { année }\end{array}$ & $\begin{array}{c}2 \mathrm{e} \\
\text { année }\end{array}$ & $\begin{array}{c}3 \mathrm{e} \\
\text { année }\end{array}$ & $\begin{array}{c}4 \mathrm{e} \\
\text { année }\end{array}$ & $\begin{array}{c}5 \mathrm{e} \\
\text { année }\end{array}$ \\
\hline Responsabilité médicale & Chi2 $=25,11 ; \mathrm{p}<0.0001$ & - & + & + & + & + \\
\hline Soin & Chi2 $=23,26 ; \mathrm{p}=0.0001$ & + & & - & - & - \\
\hline Compétence & $\mathrm{Chi} 2=17,3 ; \mathrm{p}=0.0016$ & - & - & & + & + \\
\hline Hiérarchie & $\mathrm{Chi} 2=51,47 ; \mathrm{p}<0.0001$ & - & + & + & + & \\
\hline
\end{tabular}

L'évolution de la représentation de la profession de médecin semble attester de la prise de conscience des étudiants des différences entre eutocie et pathologie qui caractérisent les relations entre sages-femmes et médecins. Des différences significatives apparaissent sur la notion de soin qui est surreprésentée en $1^{\mathrm{e}}$ année. Les notions de responsabilité médicale et de hiérarchie sont plus présentes à partir de la $2^{\mathrm{e}}$ année comme pour la profession de sage-femme. Enfin, la notion de compétence devient prégnante dès la $4 \mathrm{e}$ année. Au vu du processus représentationnel de la profession de médecin, nous pouvons faire l'hypothèse que l'image de cette profession à la fin de la formation en école ne correspond pas complètement à celle portée par les professionnelles en activité.

\section{Évolution du noyau central de La représentation de la profession de sage-femme}

Le tableau 4 rend compte des résultats obtenus au test de centralité. 
Tableau 4: Résultat du test de centralité de la représentation de la profession de sage-fermme (en pourcentages) avec le seuil donné par le test de KolmogorovSmirnov entre parenthèses. En grisé, les éléments centraux de la représentation.

\begin{tabular}{|l|c|c|c|c|c|}
\hline Item & 1e année & 2e année & 3e année & 4e année & 5e année \\
\hline $\begin{array}{l}\text { Profession } \\
\text { médicale }\end{array}$ & $98.25(91.01)$ & $93.75(75.95)$ & $90(75.17)$ & $100(75.57)$ & $90.90(76.32)$ \\
\hline $\begin{array}{l}\text { Responsabilité } \\
\text { médicale }\end{array}$ & $89.91(90.99)$ & $93.75(75.96)$ & $90(75.17)$ & $96.77(75.57)$ & $84.85(76.33)$ \\
\hline Contraintes & $89.52(91.01)$ & $93.75(75.96)$ & $93.33(75.17)$ & $96.77(75.57)$ & $90.91(76.33)$ \\
\hline Passion & $92.04(90.95)$ & $87.5(75.96)$ & $78.57(74.30)$ & $54.83(75.57)$ & $65.63(75.96)$ \\
\hline Ecoute & $96.49(90.99)$ & $96.88(75.96)$ & $100(75.17)$ & $93.55(75.57)$ & $75.76(76.33)$ \\
\hline Disponibilité & $81.53(90.87)$ & $70(75.17)$ & $90(75.17)$ & $70.97(75.57)$ & $54.55(73.33)$ \\
\hline Equipe & $81.98(90.87)$ & $75(75.96)$ & $93.33(75.17)$ & $90.32(75.57)$ & $78.79(76.33)$ \\
\hline Accouchement & $86.03(91.01)$ & $38.71(75.57)$ & $34.48(74.75)$ & $41.94(75.57)$ & $27.27(76.33)$ \\
\hline Accompagnement & $85.52(90.99)$ & $59.38(75.96)$ & $63.33(75.17)$ & $51.61(75.57)$ & $54.55(76.33)$ \\
\hline Naissance & $79.48(91.01)$ & $25(75.96)$ & $40(75.17)$ & $35.48(75.57)$ & $33.33(76.32)$ \\
\hline $\begin{array}{l}\text { Plus beau métier } \\
\text { au monde }\end{array}$ & $47.73(90.83)$ & $71.88(75.96)$ & $67.86(74.30)$ & $38.71(75.57)$ & $36.36(76.33)$ \\
\hline Nouveau-né & $76.96(91.03)$ & $19.35(75.57)$ & $37.93(74.75)$ & $32.26(75.57)$ & $27.27(76.33)$ \\
\hline Grossesse & $84.44(90.93)$ & $65.63(75.96)$ & $53.33(75.17)$ & $61.29(75.57)$ & $36.36(76.33)$ \\
\hline
\end{tabular}

Nous pouvons observer la stabilité de certains éléments du noyau central entre les années de formation: la notion de profession médicale est centrale pour toutes les années, celle de responsabilité médicale et de contraintes le deviennent dès la première année en école et le restent jusqu'à la fin. Le statut d'autres items évolue au fur et à mesure de la progression dans les études: passion est central jusqu'en $3^{\mathrm{e}}$ année, écoute jusqu'en $4^{\mathrm{e}}$ année, équipe devient central à partir de la $3^{\mathrm{e}}$ année. Enfin, la notion de disponibilité n'apparaît centrale que lors de la $3^{\mathrm{e}}$ année. Nous noterons également que les notions de naissance, de nouveau-né et d'accouchement perdent de de l'importance au cours de l'avancement dans la formation. Ces résultats viennent renforcer les analyses précédentes.

\section{Discussion}

Au vu des différents éléments évoqués, nous pouvons dire qu'il existe dans cet échantillon des transformations remarquables au niveau du noyau central et de la périphérie de la représentation de la profession de sage-femme durant la formation ainsi qu'une évolution de la représentation de la profession de médecin.

Ces résultats nous permettent de discuter quelques points autour de la formation des sages-femmes, en gardant à l'esprit l'existence dans ce système d'une alternance entre cours et stages. Le stress apparaît dans les représentations des étudiants dès le début de la formation (Tableau 2) et transparaissait 
déjà lors de notre première enquête auprès des acteurs de la périnatalité. Notre expérience de formatrice en école de sages-femmes nous permet d'affirmer que cette notion est trop peu prise en compte. Il pourrait être intéressant de mettre en place des séances de retours de stage ou de vécu d'expérience ciblées sur cette thématique. Concrètement, ce pourrait être réalisé sous forme d'atelier d'écriture (Balcou-Debussche, 2005).

La notion de contraintes est apparue au cours de cette étude (Tableau 4), alors qu'elle était apparue chez les professionnelles uniquement lors de la méthode de substitution, comme un élément d'une zone muette (Flament, Guimelli \& Abric, 2006, p.16). Les étudiants prennent conscience de l'ensemble des contraintes de la profession que ce soit au niveau de la formation, de la hiérarchie ou de l'exercice professionnel. C'est peut-être la présence des deux objets dans un même questionnaire qui est à l'origine de la sur représentation des contraintes. Comme le suggère les travaux de Tajfel (1972) la présence d'un autre groupe dans le questionnaire a pu suractiver le processus de différenciation intergroupe. Le terme de contraintes peut également être lié au contexte de revendication (les sages-femmes étaient en grève sur le plan national au moment de cette enquête) qui pourrait participer à survaloriser cet élément. Cette notion de contraintes est un élément à prendre en compte dans les formations en école de sagesfemmes, mais également dans l'organisation même des services. Même si le suivi individuel permet à l'étudiant d'exprimer celles qu'il perçoit et ainsi de pouvoir adapter son projet professionnel (par exemple par le choix du lieu de stage en $5^{\mathrm{e}}$ année), il nous semble que seul un changement des conditions réelles d'exercice peuvent participer à limiter la perception de ces contraintes.

L'autonomie est spécifique de la $5^{\mathrm{e}}$ année (Tableau 2) qui est quasi professionnelle et qui se détache de certaines préoccupations des années précédentes pour se recentrer sur l'essentiel de la profession (versant technique). L'autonomie est en effet un élément prégnant dans l'évolution du processus de professionnalisation, élément que nous retrouvons par ailleurs dans les représentations professionnelles de la profession de sage-femme auprès des sages-femmes. Nous pouvons rapprocher ces éléments de la notion de «entiment de contrôle du modèle Sens-Repères-Contrôle de l'implication professionnel de Mias (1998). Il nous apparaît comme un marqueur d'une implication active, signe de professionnalisation, d'étudiants qui ont conscience qu'ils devront très prochainement agir en toute autonomie. Cette prise de conscience doit être accompagnée de façon bienveillante car un des enjeux du formateur est de faire surmonter l'angoisse et le stress inhérents à cette autonomie. Les groupes de parole sont aussi un lieu d'expression et de facilitation (Robert, 2008).

L'équipe, notion absente chez les professionnelles ou faisant partie de l'implicite professionnel, apparaît de manière significative au travers de l'utilisation de termes évoquant l'ensemble de la profession médicale comme par exemple l'utilisation du terme de gynécologues obstétriciens (Tableau 4). Il semble donc pertinent, dans les travaux dirigés d'exploitation de dossiers 
médicaux, de faire transparaître la collaboration interdisciplinaire auprès des patientes.

L'expression le plus beau métier du monde, présente chez les professionnelles, n'est prégnante qu'en $2^{\mathrm{e}}$ année de formation: elle conforte les étudiants dans le choix de la formation lors de l'entrée en école et permet sans doute de rationaliser les difficultés rencontrées (Tableau 4). Cet élément est absent dans l'expression des représentations de la profession de sage-femme au cours du cursus de formation. Les étudiants se concentrent donc au cours de la formation sur leurs apprentissages propres de la profession de sage-femme. Le formateur doit cependant veiller à ce que la motivation reste un élément moteur de leur formation.

La responsabilité médicale apparaît dès la $2^{\mathrm{e}}$ année de formation et peut être une piste à explorer pour commencer un enseignement spécifique sur la législation professionnelle de la profession de sage-femme et les compétences inhérentes à la profession. Nous pourrions faire l'hypothèse que l'obtention du concours, l'impact de la $1^{\mathrm{e}}$ année ou les premiers moments en école induisent l'apparition de ce terme. Cette prise de conscience est nécessaire à l'enseignement des unités spécifiques car la responsabilité médicale est un élément prégnant de la représentation de la profession de sage-femme, à la fois chez les sages-femmes et chez les médecins.

\section{Conclusion}

Il existe bien un «effet formation» sur les représentations des deux professions: il nous semble que ces résultats attestent de la structuration chez ces étudiants d'un discours professionnel sur ces deux objets. Une évolution de la notion d'accouchement vers la notion d'accompagnement en fin de cursus pour la profession de sage-femme ainsi que celle du soin vers la maladie pour la profession de médecin témoigne par exemple de cet effet. Les aspects spécifiques de chacune des professions transparaissent: la physiologie pour les sages-femmes et la pathologie pour les médecins, ainsi que le passage d'un système d'apprentissage à l'autonomie. On observe le passage d'une représentation naïve de la profession de sage-femme en $1^{\mathrm{e}}$ année avec les termes d'accouchement et de nouveau-né vers une profession médicale à responsabilités en fin de cursus. Il existe alors un réel intérêt de réinterroger les processus de formation.

Le processus de professionnalisation est aussi lié à l'alternance et l'influence de changements de pratiques avec la réforme actuelle du programme des études aura sûrement une influence sur l'évolution des représentations de la profession de sage-femme et inversement: aux professionnels enseignants de faire les bons choix pour ouvrir les perspectives vers une perception moindre des contraintes.

L'approche structurale des représentations de la profession de sage-femme et de la profession de médecin permet de rendre compte qu'à partir d'éléments 
similaires, deux représentations peuvent se construire différemment et être porteuses de sens différents. Une étude longitudinale auprès des étudiants et des jeunes professionnels sages-femmes pourrait compléter cette étude transversale sur l'évolution de ce processus représentationnel et permettrait ainsi de mieux appréhender (ou plus justement) la transformation-professionnalisation qui s'opère au cours de cette formation. Une telle étude permettrait par ailleurs de dépasser la limite du faible échantillon que nous avons interrogé et de mettre éventuellement en évidence des variations sur ces représentations dues à des ancrages culturels différents des équipes de formation intervenant dans les 35 écoles de sages-femmes en France.

Cette prise de conscience de l'évolution du mode de perception de la profession de sage-femme et de la profession de médecin chez les étudiants sagesfemmes permet d'adapter certaines unités d'enseignement théoriques à certains temps de la formation mais aussi certains temps de stage comme nous avons pu le constater. Ces adaptations sont aussi des temps nécessaires au processus de professionnalisation.

\section{Bibliographie}

Abric, J.-C. (1987). Coopération, compétition et représentations sociales. Cousset: Delval.

Abric, J.-C. (2003). Pratiques sociales et représentations. Paris: PUF.

Abric, J.-C. (Éd.). (2005). Les Représentations sociales: méthodes d'étude des représentations sociales. Ramonville St-Agne: Eres.

Balcou-Debussche, M. (2005). L'écriture et la socialisation professionnelle des étudiants: l'exemple des infirmiers, sages-femmes et aides-soignants en formation initiale. Le sociographe, 18, 47-58.

Bataille, M. (2000). Représentation, implicitation, implication. In C. Garnier \& M-L. Rouquette (Éd.), Représentations sociales et éducation (pp. 165-189). Montréal, Québec: Éditions nouvelles.

Bataille, M. (2007). Qu'est-ce que vieillir pour un chercheur? In C. Montandon \& J. Trinc (Éd.), Vieillir dans le métier (pp. 245-250). Paris: L'Harmattan.

Blin J.-F. (1997). Représentations, pratiques et identités professionnelles. Paris: L'Harmattan.

Bodin, R. \& Orange, S. (2013). L'université n'est pas en crise. Les transformations de l'enseignement supérieur: enjeux et idées reçues. Bellecombe-en-Bauges: Éditions du Croquant.

Degenne, A. \& Vergès, P. (1973). Introduction à l'analyse de similitude. Revue française de sociologie, 14, 471-511.

Flament, C. (1962). L'analyse de similitude. Cahiers du centre de recherche opérationnelle, 4, 63-97.

Flament, C., Guimelli, C. \& Abric, J.-C. (2006). Effets de masquage dans l'expression d'une représentation sociale. Cahiers internationaux de psychologie sociale, 69(1), $15-32$.

Fraysse, B. (1996). Évolution des représentations socioprofessionnelles des élèves ingénieurs: étude diachronique comparative sur trois départements de l'INSA de Toulouse. Thèse en Sciences de l'Éducation, Université Toulouse Jean Jaurès.

Frégonèse, C. \& Ratinaud, P. (2015). Connaissance des représentations professionnelles de la profession de sage-femme. La Revue Sage-Femme, 14(1), 7-13.

Gachassin, B., Labbé, S. \& Mias, C. (2013). Les étudiants face à la professionnalisation à l'université. Recherche et formation, 73, 37-56. 
Gayraud, L., Simon-Zarca, G. \& Soldano, C. (2011). Université: les défis de la professionnalisation. Notes Emploi Formation, 46(5), 1-36.

Jodelet, D. (1991). Représentation sociale. In H. Bloch, R. Chemama \& E. Dépret (Éd.), Grand Dictionnaire de la psychologie (p. 668-672). Paris: Larousse.

Lac, M. (2003). Un groupe en formation: contribution à l'analyse des transformations de l'implication et des représentations: l'exemple du DEUST «médiation sociale, éducative et documentaire: les métiers de l'animation». Thèse de Doctorat en Sciences de l'Éducation et de la Formation, Université Toulouse Jean-Jaurès.

Lac, M., Mias, C., Labbé, S. \& Bataille, M. (2010). Les représentations professionnelles et l'implication professionnelle comme modèles d'intelligibilité des processus de professionnalisation. Les dossiers des Sciences de l'Éducation, 24, 133-145.

Lo Monaco, G., Lheureux, F. \& Halimi-Falkowicz, S. (2008). Test d'indépendance au contexte (TIC) et structure des représentations sociales. Swiss Journal of Psychology / Revue Suisse de Psychologie, 67(2), 119-123.

Maubant, P., Roger, L., Caselles-Desjardins, B., Mercier, B. \& Gravel, N. (2010). Questions et enjeux dans l'étude compréhensive des processus de professionnalisation dans les métiers de l'intervention humaine. Les dossiers des Sciences de l'Éducation, 24, 109-132.

Mias, C. (1998). L'implication professionnelle dans le travail social. Paris: L'Harmattan.

Michalot, T. \& Simeone, A. (2013). Formation et évolution des représentations professionnelles: le cas des critères d'accueil de travailleurs sociaux en CHRS. Recherche et formation, 72, 133-146.

Moliner, P. (Éd.). (2001). La dynamique des représentations sociales. Grenoble: PUG.

Moliner, P., Rateau, P. \& Cohen-Scali, V. (2002). Les représentations sociales: pratique des études de terrain. Rennes: Presses Universitaires de Rennes.

Moscovici, S. (1976). La psychanalyse son image et son public. Paris: PUF.

Piaser, A. (1999). Représentations professionnelles à l'école: Particularités selon le statut: enseignant, inspecteur. Thèse de Doctorat en Sciences de l'Éducation et de la Formation, Université Toulousejean-Jaurès.

Piaser, A. \& Bataille, M. (2011). Of contextualised use of «social» and «professional». In M. Chaïb, B. Danermark \& S. Selander (Éd.), Education, Professionnalization and Social Representations. On the Transformation of Social Knowledge (pp. 44-54). New-York, Oxon: Routlegde.

Piaser, A. \& Ratinaud, P. (2010). Pensée sociale, pensée professionnelle: une approche singulière en Sciences de l'Éducation. Les Dossiers des Sciences de l'Éducation, 23, 7-14.

R Development Core Team. (2014). R: A language and environment for statistical computing. R Foundation for Statistical Computing. Vienna, Austria. Consulté le 15 janvier dans http://www.R-project.org

Ratinaud, P. (2003). Les professeurs et Internet: Contribution à la modélisation des pensées sociale et professionnelle par l'étude de la représentation professionnelle d'Internet. Thèse de Doctorat en Sciences de l'Éducation et de la Formation, Université Toulouse Jean-Jaurès.

Ratinaud, P. (2014). IRaMuTeQ: Interface de R pour les Analyses Multidimensionnelles de Textes et de Questionnaires (Version 0.7 alpha 2). Consulté le 8 janvier 2016 dans http://www. iramuteq.org

Ratinaud, P. \& Lac, M. (2011). Understanding professionalization as a representational process. In M. Chaïb, B. Danemark \& S. Selander (Éd.), Education, Professionnalization and Social Representations. On the Transformation of Social Knowledge (pp. 55-67). New-York, Oxon: Routlegde.

Remoussenard, P. (2010). Des liens entre besoin de connaissance du travail et perspectives de professionnalisation. Les dossiers des Sciences de l'Éducation, 24, 13-27.

Robert, P. (2008). Groupes de parole pour formation de soignants. Revue de psychothérapie psychanalytique de groupe, 50, 133-139. 
Tajfel, H. (1972). La catégorisation sociale. In S. Moscovici (Éd.), Introduction à la psychologie sociale (pp. 272-299). Paris: Larousse.

Wittorski, R. (2007). Professionnalisation et développement des compétences professionnelles. Paris: L'Harmattan.

Wittoski, R. (2008). La professionnalisation. Savoirs, 17, 11-38. Consulté le 20 mai 2015 2016 dans http://halshs.archives-ouvertes.fr

Wittorski, R. (2010). La professionnalisation: d'un objet social à un objet scientifique. Les dossiers des Sciences de l'Éducation, 24, 7-11.

Wittorski, R. (2012). La professionnalisation de l'offre de formation universitaire: quelques spécificités. Revue internationale de pédagogie de l'enseignement supérieur, 28(1), 1-13.

Mots-clés: Professionnalisation, représentations sociales et professionnelles, formation initiale par alternance, étudiants sages-femmes

\section{Die Professionalisierung der Geburtshilfe: dynamische Analyse der sozialen und beruflichen Vorstellungen.}

\section{Zusammenfassung}

Diese Forschung konzentriert sich auf die Dynamik der sozialen und beruflichen Vorstellungen von Studierenden der Geburtshilfe im Prozess der Professionalisierung. Basierend auf Theorien zu sozialen und berufsbezogenen Einstellungen steht die Wahrnehmung zweier Berufsgruppen im Mittelpunkt: der Hebammenberuf und der Ärzteberuf.

In einer Querschnitts-Studie wurden 358 Studierende $\mathrm{zu}$ verschiedenen Zeitpunkten (vor der Zulassung bis ins vierte Ausbildungsjahr) mittels Fragebogen befragt. Die Ergebnisse zeigen, dass sich die Vorstellungen der Studierenden während der Ausbildung verändern.

Schlagworte: Professionalisierung, soziale und berufliche Vorstellungen, duale Ausbildung, Hebammenausbildung 


\section{La professionalizzazione delle ostetriche: analisi dinamica delle rappresentazioni sociali e professionali.}

\section{Riassunto}

Questa ricerca si concentra sulle dinamiche di rappresentazione sociale e professionale coinvolte nel processo di professionalizzazione degli studenti di ostetricia. Basandosi sulla teoria delle rappresentazioni sociali e professionali, interroga un sistema di rappresentazioni costituito da due oggetti: la professione ostetrica e la professione medica.

Uno studio trasversale tramite questionari con 358 studenti in diversi momenti della formazione (dall'ammissione al quarto anno di scuola) ha messo in evidenza l'evoluzione di queste rappresentazioni durante la formazione.

Parole-chiave: Professionalizzazione, rappresentazioni sociali e professionali, formazione iniziale in alternanza, studenti ostetriche

\section{Midwives' professionalization: analyse with social and professional representations dynamics.}

\section{Summary}

This research focuses on the dynamics of social and professional representations involved in the process of professionalization of midwifery students. It mobilizes the theory of social and professional representations and asks a representational system of two objects: the profession of midwifes and the profession of doctor.

A transverse study by questionnaires with 358 students in different stages of their training (a total of 5 years, from the year of the exam to the fourth year in midwives' school) highlighted the evolution of these representations during training.

Keywords: Professionnalization, social and professionnal representations, initial work-linked training, midwifery students 
